\title{
Overexpression of miR-874-3p alleviates LPS-induced apoptosis and inflammation in alveolar epithelial cell by targeting EGR3/NF-KB
}

\author{
Huirun Yang', Yang Dong², Yan Zhou ${ }^{3}$ and Huajun Li4⿴囗十 \\ 1Pediatrics of Integrated Traditional Chinese \& Western Medicine, Zhejiang Zhoushan Putuo District Hospital of Traditional Chinese Medicine, \\ Zhoushan, Zhejiang Province, 316100, China; 2Department of Pediatrics, People's Hospital of Baoshan City, Baoshan, Yunnan Province, 678000, \\ China; ${ }^{3}$ Department of Pediatrics, People's Hospital of Baiyun District, Guiyang City, Guizhou Province, 510440, China; ${ }^{4}$ Department of Infection, \\ Xinhua Hospital Affiliated to Shanghai Jiao Tong University School of Medicine, Shanghai, 200092, China
}

Objective: MicroRNA (miRNA) is implicated in the pathogenic mechanism of pneumonia. Role of miR-874-3p in pediatric pneumonia was therefore evaluated in this study. Methods: Expression levels of miR-874-3p in the serum samples from pediatric patients with pneumonia and LPS-treated HPAEpiC were determined by RT-qPCR (reverse transcription quantitative real-time PCR). Secretion of inflammatory factors in LPS-treated HPAEpiC were determined by qRT-PCR and ELISA. Cell viability and apoptosis were evaluated by CCK8 and flow cytometry, respectively. HPAEpiC was used for the validation of binding target of miR-874-3p. Mechanism was determined by NF-KB promoter activity assay. Results: MiR-874-3p was reduced in serum samples of pediatric patients with pneumonia, and LPS treatment dose-dependently decreased miR-874-3p expression in HPAEpiC. TNF- $\alpha$ and IL-1 $\beta$ expression levels were increased in HPAEpiC post LPS treatment. Over-expression of miR874-3p attenuated LPS-induced increase of TNF- $a$ and IL-1 $\beta$ and reversed LPS-induced decrease of cell viability and increase of cell apoptosis in HPAEpiC. EGR3 (early growth response 3), increased in LPS-induced HPAEpiC, was a target gene of miR-874-3p. EGR3 over-expression reversed miR-874-3p over-expression-induced increase of cell viability, decrease of cell apoptosis, TNF- $a$ and IL$1 \beta$ in LPS-induced HPAEpiC. Over-expression of miR-874$3 p$ reduced $p 65$ expression and NF-KB promoter activity in LPS-induced HPAEpiC, while EGR3 over-expression reversed these suppressive effects. Conclusion: MiR-874-3p negatively regulates EGR3 expression to promote cell viability and inhibit apoptosis as well as inflammation in LPS-treated HPAEpiC via suppression of NF-KB pathway, suggesting a potential therapeutic strategy for pneumonia.

Keywords: miR-874-3p, EGR3, LPS, HPAEpiC, pediatric pneumonia

Received: 19 October, 2020; revised: 02 December, 2020; accepted: 14 December, 2020; available on-line: 26 May, 2021

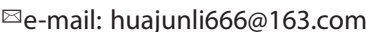

Abbreviations: MiRNA, microRNA; EGR3, early growth response 3 ; HPAEpiC, human pulmonary alveolar epithelial cell; RT-qPCR, reverse transcription quantitative real-time PCR

\section{INTRODUCTION}

Pneumonia is a common and frequently occurring respiratory disease in pediatrics. It is one of the three most important pediatric diseases and the leading cause of infant mortality worldwide (Alcoba et al., 2017). Pneu- monia is associated with pathogenic factors post infection with bacteria or viruses, and microbial pathogensinduced stimulation of inflammatory response was considered as one of the causes of severe acute pediatrics pneumonia (Qinghe et al., 2019; Zhang et al., 2019). Alveolar epithelial cells are the major targets of inflammatory factors secreted by the infected epithelial cells during the inflammatory response in the lungs (Ito et al., 2015). Damage to alveolar epithelial cells is a key step in the development of pneumonia and ultimately leads to fluid imbalance (Guillot et al., 2013). The state of alveolar epithelial cells contributes to the loss of cellular defense during development of pneumonia (Robb et al., 2016). Therefore, it is important to reduce the inflammation and apoptosis of alveolar epithelial cells during the treatment of pediatric pneumonia.

Dysregulation of microRNAs (miRNAs) has been identified as diagnostic biomarkers for pneumonia (Huang et al., 2017), and miRNAs could mediate expression of target genes involved in immune response during pneumonia (Huang et al., 2018). Therefore, miRNAs were widely investigated in pediatric pneumonia. Recently, miRNAs have been reported to be implicated in apoptosis and inflammation of alveolar epithelial cells (Li \& Liu, 2020). Suppression of apoptosis and inflammation in alveolar epithelial cells by miR-424 could ameliorate acute respiratory distress syndrome (Cheng et al., 2020). MiR-874-3p was reported to increase cell proliferation and suppress apoptosis during cerebral ischemia/reperfusion injury (Jiang et al., 2019), and inhibit inflammatory response during ischemic stroke (Xie et al., 2020). Given that the expression of miR-874-3p was down-regulated in patients with pneumonia in this study, we speculated that miR-874-3p might regulate apoptosis and inflammation of alveolar epithelial cells and play a certain regulatory role in pneumonia development.

EGR3 (early growth response 3) functions as a transcriptional factor to regulate genes involved in inflammation (Xie et al., 2020), cell proliferation ( $\mathrm{Li}$ et al., 2012) and differentiation (Miao et al., 2017). EGR1 pathway was involved in lung inflammation (Cho et al., 2006), and epithelial barrier disruption (Choi et al., 2012). However, no researches about regulation of EGR in inflammation during pneumonia have not been reported yet. This study was performed to investigate whether miR-874-3p could target EGR3 to regulate apoptosis and inflammation of alveolar epithelial cells. 


\section{MATERIALS AND METHODS}

\section{Serum collection}

This study was approved by the Ethics Committee of Zhejiang Zhoushan Putuo District Hospital of Traditional Chinese Medicine. Twenty-seven pediatric patients with pneumonia and nineteen healthy children were recruited at Zhejiang Zhoushan Putuo District Hospital of Traditional Chinese Medicine with written parental informed consents. Patients with anti-inflammatory therapy were excluded from this study, and patients with diagnostic criteria of pneumonia were included in this study. Serum samples were obtained from pneumonia patients and healthy children and stored at $-80^{\circ} \mathrm{C}$ for the functional assays.

\section{CELL CULTURE, TREATMENT AND TRANSFECTION}

Human pulmonary alveolar epithelial cell (HPAEpiC; ATCC; Manassas, VA, USA) was cultured in RPMI-1640 medium with high glucose (Lonza, Basel, Switzerland) containing 10\% fetal bovine serum (Gibco, Waltham, $\mathrm{MA}, \mathrm{USA})$ at $37^{\circ} \mathrm{C}$. For cell transfection, mimic and inhibitor of miR-874-3p and negative controls (NC mimic and NC inhibitor) were purchased from GenePharma (Shanghai, China). Full length CDS of EGR3 was inserted into pcDNA3.1 vector (Invitrogen, Carlsbad, CA, USA). Transfection was then performed via Lipofectamine 2000 (Invitrogen) into HPAEpiC. Two days after transfection, HPAEpiC was incubated in RPMI1640 medium with high glucose containing 10\% fetal bovine serum and 1, 10 or $50 \mathrm{mg} / \mathrm{L} \mathrm{LPS} \mathrm{(Sigma} \mathrm{Al-}$ drich, St. Louis, MO, USA) for 48 hours before functional assays.

\section{Detection of TNF- $\alpha$ and IL-1 $\beta$}

Supernatant was harvested from medium of cell culture through centrifugation at $400 \times g$ for 10 minutes. Commercial Elisa kits (Thermo Fisher, Waltham, MA, USA) were used for the detection of TNF- $\alpha$ and IL-1 $\beta$.

\section{Cell viability and apoptosis assays}

HPAEpiC with indicated treatment was seeded for 24 hours followed by LPS incubation. CCK8 solution (Dojindo, Tokyo, Japan) was then added to the wells and incubated at $37^{\circ} \mathrm{C}$ for 1 hour. Absorbance at $450 \mathrm{~nm}$ was measured by Microplate Autoreader (Thermo Fisher). HPAEpiC with indicated treatment and transfection were harvested and resuspended in binding buffer (Invitrogen) with PI $(100 \mu \mathrm{g} / \mathrm{mL})$ and $1 \mathrm{U} / \mathrm{ml}$ ribonuclease (Invitrogen) for 30 minutes, and then incubated with fluorescein isothiocyanate-conjugated annexin V (20 $\mu \mathrm{g} /$ $\mathrm{mL}$ ) for another 20 minutes. Apoptosis was analyzed by FACS flow cytometer (Attune, Life Technologies, Darmstadt, Germany).

\section{Luciferase reporter assay}

3'-UTR of EGR3 or sequence with mutation at the potential binding site between EGR3 and miR-874-3p were subcloned into pGL3 luciferase vector (Promega, Madison, Wisconsin, USA). Co-transfection with EGR3WT/EGR3-MUT or mimic and inhibitor of miR-874-3p were performed through Lipofectamine 2000. Dual Luciferase Assay Kit (Promega) was used to detect luciferase activities two days later.

\section{NF-KB promoter activity}

HPAEpiC were co-transfected with NF- $x$ B-Luc plasmid (Promega) and pRT-TK (Promega) with either miR874-3p mimic or pcDNA-EGR3 and miR-874-3p mimic. Dual Luciferase Assay Kit was then used to detect the activities two days later.

\section{RT-qPCR (reverse transcription quantitative real-time PCR)}

Total RNAs were isolated from serum samples or HPAEpiC by Trizol (Invitrogen), and reverse-transcribed into cDNAs via PrimeScript RT Master Mix kit (Takara Biotechnology, Dalian, China). TB Green Premix Ex Taq (Takara, Dalian, China) was applied for qRT-PCR analysis. Caenorhabditis elegans miRNA (Cel-miR-39) was used as control to analyze miR-874-3p expression from serum samples. U6 was used as endogenous control to analyze miR-874-3p expression from cells. GAPDH was used as endogenous control to analyze TNF- $\alpha$, IL-1 $\beta$ and EGR3 expression. Threshold cycle (Ct) value was determined to reflect fluorescent signal of amplification plot, and the relative gene expression was normalized to the controls by the $2^{-\triangle \Delta C T}$ method. The primer sequences were shown as below:

Primer table

\begin{tabular}{ll}
\hline ID & Sequence (5'- 3') \\
\hline GAPDH F & ACCACAGTCCATGCCATCAC \\
\hline GAPDH R & TCCACCACCCTGTTGCTGTA \\
\hline TNF- $\alpha$ F & ACTGAACTTCGGGGTGATTG \\
\hline TNF-a R & GCTTGGTGGTTGCTACGAC \\
\hline IL-1 $\beta$ F & CACCTTCTTTCCTTCATCTTT \\
\hline IL-1 $\beta$ R & GTCGTTGCTTGTCTCTCCTTGTA \\
\hdashline miR-874-3p F & GGCCCTGAGGAAGAACTGAG \\
\hline miR-874-3p R & TGAGATCCAACAGGCCTTGAC \\
\hline EGR3 F & CGAGGACAAAAGCGTCGAAGCTC \\
\hline EGR3 R & GATCAAGGCGATCCTAACTGAAC \\
\hline Cel-miR-39 F & CAGAGTAGCTCACCGGGTGTAAATC \\
\hline Cel-miR-39 R & CCAGTGCAGGGTCCGAGGTAT \\
\hline
\end{tabular}

\section{Western blot}

Total proteins were collected from HPAEpiC by radioimmunoprecipitation assay buffer (Beyotime Institute of Biotechnology, Beijing, China). Proteins (30 $\mu \mathrm{g})$ were separated via SDS-PAGE, then transferred 
to PVDF membrane. Membranes were blocked and incubated with the following primary antibodies: antiEGR3 (1:2000; Cell Signaling Technology, Danvers, MA, USA), anti-Bcl-2 (1:2000; Cell Signaling Technology), anti-Bax (1:2500; Cell Signaling Technology), anti-cleaved caspase-3 (1:2500; Cell Signaling Technology), p65 (1:3000; Cell Signaling Technology) and anti- $\beta$-actin (1:3000; Cell Signaling Technology). After incubation with second antibodies (1:5000; Cell Signaling Technology) for 1 hour, the membranes were conducted with ECL chemiluminescence detection kit (Thermo Fisher), and the blots were visualized and quantified via Quantity One 4.6.2 software (Bio-Rad, Hercules, CA, USA).

\section{Statistical analysis}

Data were expressed as mean \pm standard deviation. Statistical analyses between different groups were analyzed by GraphPad Prism 5.0 and determined by oneway analysis of variance and Student's $t$-test. $p<0.05$ was considered as statistically significant.

\section{RESULTS}

\section{Reduced miR-874-3p in serum of pediatric patients with pneumonia}

To unravel potential role of miR-874-3p in pediatric pneumonia, serum samples were collected from patients with pneumonia. Data from qRT-PCR demonstrated that miR-874-3p was reduced in serum of pediatric patients with pneumonia compared to the healthy
A

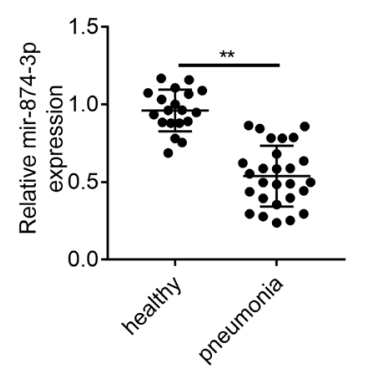

B

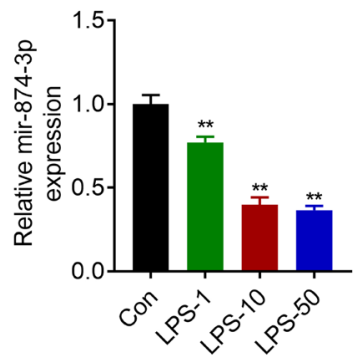

Figure 1. Reduced miR-874-3p in serum of pediatric patients with pneumonia

(A) Down-regulation of miR-874-3p in serum of patients with pneumonia compared to the healthy control. (B) Down-regulation of miR-874-3p in HPAEpiC post treatment with different concentration of LPS. ${ }^{* *} p<0.01$.

control (Fig. 1A). The relationship between miR-874-3p expression and clinical characteristics of sick children was shown in Table 1. Symptom and signs, including cough, expectoration, dyspnea, and diarrhea, as well as laboratory tests, including white blood cells, neutrophils, and lymphocytes, were significantly associated with miR-874-3p expression (Table 1). HPAEpiC with LPS treatment was used to establish in vitro cell model of pneumonia. A dose-dependent reduction of miR874-3p was identified in LPS-treated HPAEpiC (Fig. $1 B)$, suggesting a potential role of miR-874-3p in pediatric pneumonia.

Table 1. Association between miR-874-3p expression and clinico-pathological parameters.

\begin{tabular}{|c|c|c|c|c|}
\hline \multirow{2}{*}{ Characteristics } & \multirow{2}{*}{ Number of patients } & mir-874-3p & mir-874-3p & \multirow{2}{*}{$P$ value } \\
\hline & & Low expression ( $\leq$ medin) & High expression (> medin) & \\
\hline Number & 46 & 27 & 19 & \\
\hline Gender & & & & 0.446 \\
\hline Male & 26 & 14 & 12 & \\
\hline Female & 20 & 13 & 7 & \\
\hline Ages (years) & & & & 0.806 \\
\hline$<10$ & 30 & 18 & 12 & \\
\hline$\geq 10$ & 16 & 9 & 7 & \\
\hline \multicolumn{5}{|l|}{ Symptom and signs } \\
\hline Cough & 29 & $25(92.59 \%)$ & $4(21.05 \%)$ & $p<0.001$ \\
\hline Expectoration & 19 & $17(62.96 \%)$ & $2(10.53 \%)$ & $p<0.001$ \\
\hline Dyspnea & 10 & $9(33.33 \%)$ & $1(5.26)$ & 0.023 \\
\hline Diarrhea & 12 & $10(37.04 \%)$ & $2(10.53 \%)$ & 0.044 \\
\hline \multicolumn{5}{|l|}{ Laboratory tests } \\
\hline White blood cell $\left(\times 10^{9} / L\right)$ & & $11.9 \pm 1.4$ & $10.27 \pm 1.97$ & 0.002 \\
\hline Neutrophils (\%) & & $53.2 \pm 12.17$ & $38.9 \pm 10.23$ & $p<0.001$ \\
\hline Lymphocyte (\%) & & $43.8 \pm 17.12$ & $59.12 \pm 18.23$ & 0.005 \\
\hline
\end{tabular}




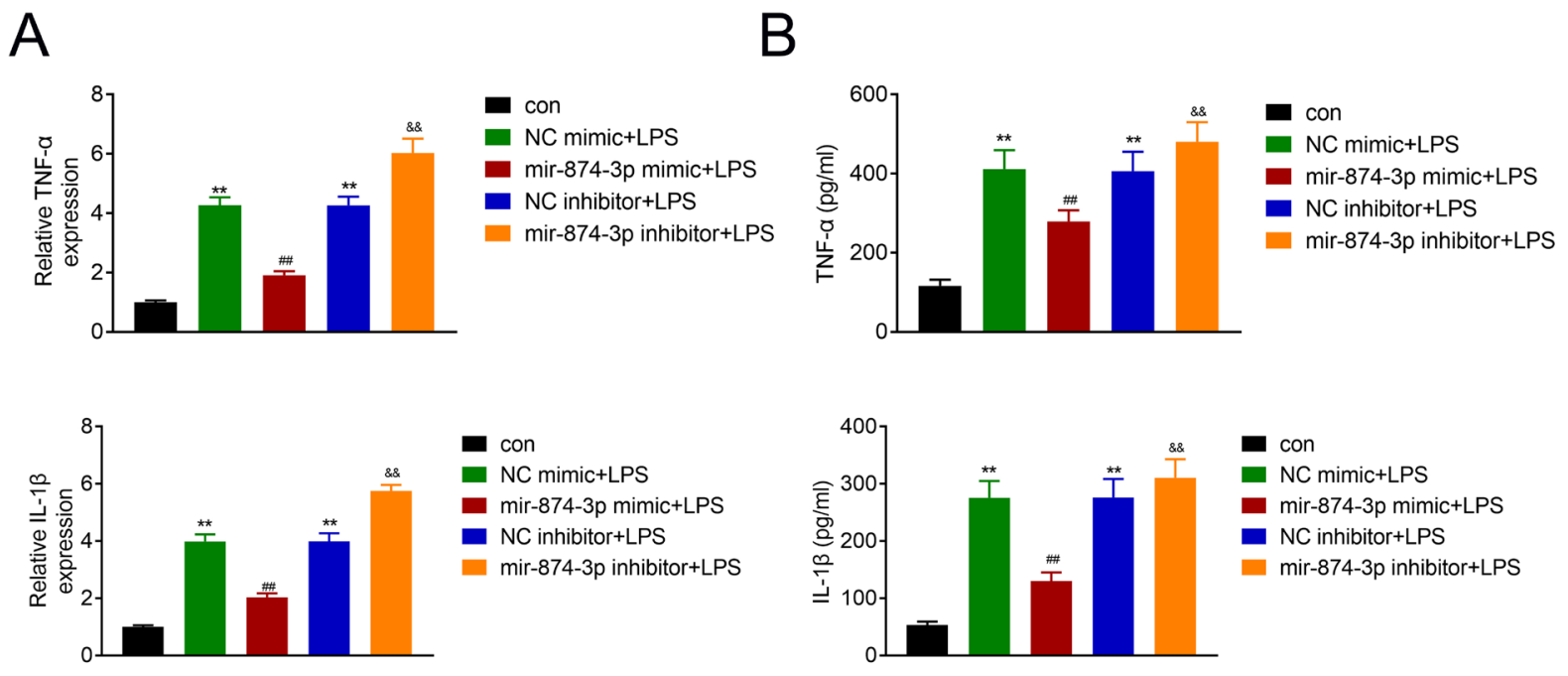

Figure 2. MiR-874-3p attenuated LPS-induced inflammation in HPAEpiC

(A) Transfection with miR-874-3p mimic decreased mRNA expression of TNF-a and IL-1 $\beta$ in LPS-treated HPAEpiC, while miR-874-3p inhibitor transfection increased the expression. (B) Transfection with miR-874-3p mimic decreased protein expression of TNF-a and IL-1 $\beta$ in

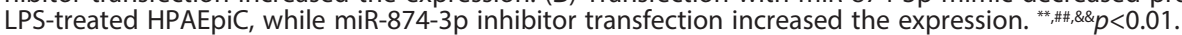

A

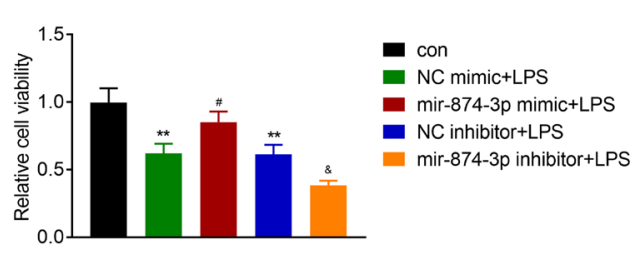

B

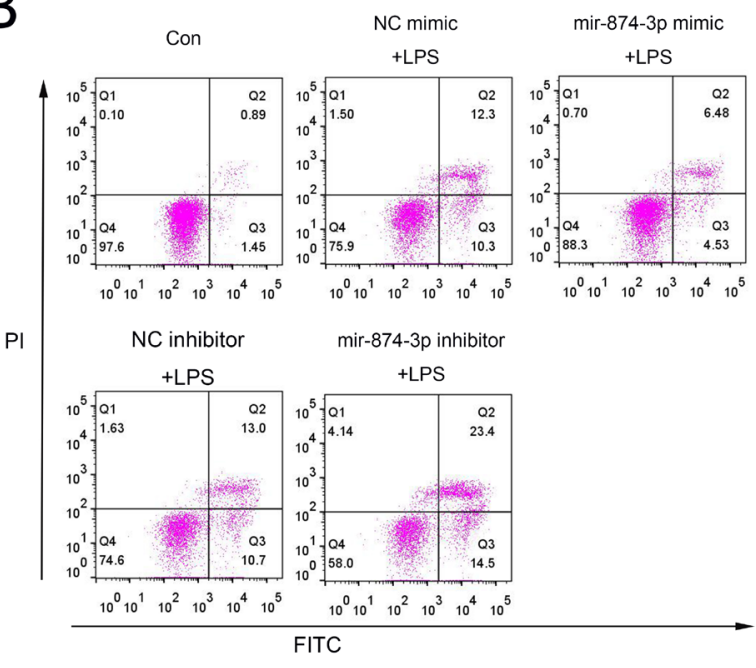

C

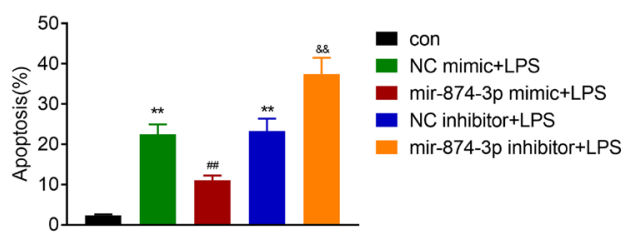

Dir-874-3p inhibitor $\quad-\quad-\quad+$

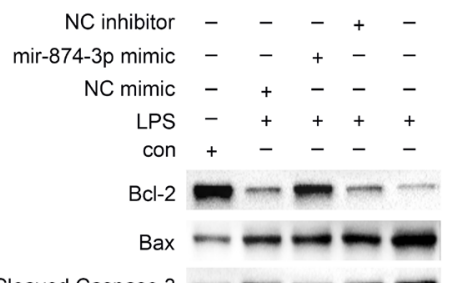

Cleaved Caspase $3 \longrightarrow \rightarrow$

$\beta$-actin

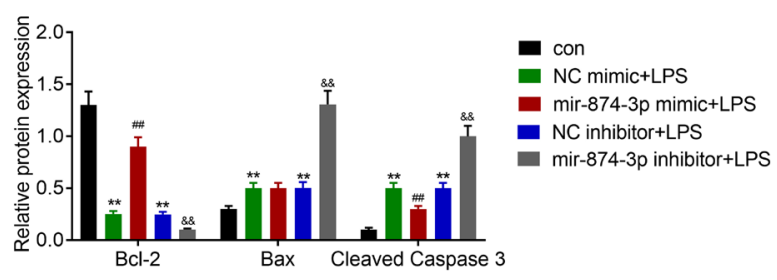

Figure 3. MiR-874-3p attenuated LPS-induced apoptosis in HPAEpiC

(A) Transfection with miR-874-3p mimic increased viability of LPS-treated HPAEpiC, while miR-874-3p inhibitor transfection decreased the cell viability. (B) Transfection with miR-874-3p mimic decreased apoptosis of LPS-treated HPAEpiC, while miR-874-3p inhibitor transfection increased apoptosis. (C) The apoptosis rate affected by miR-874-3p in LPS-treated HPAEpiC. (D) Transfection with miR-874-3p mimic increased $\mathrm{Bcl}-2$ expression while decreased Bax and cleaved caspase-3 in LPS-treated HPAEpiC, while miR-874-3p inhibitor transfection reversed the effects. \& $p<0.05, * * \#, \& \& p<0.01$.

\section{MiR-874-3p attenuated LPS-induced inflammation in HPAEpiC}

HPAEpiC was transfected with miR-874-3p mimic or inhibitor, and then incubated with $10 \mathrm{mg} / \mathrm{L}$ LPS for the evaluation of the role of $\mathrm{miR}-874-3 \mathrm{p}$ in pneu- monia. LPS stimulated inflammation in HPAEpiC with increased mRNA (Fig. 2A) and protein (Fig. 2B) expression of $T N F-\alpha$ and IL-1 $\beta$. However, transfection with miR-874-3p in LPS-treated HPAEpiC decreased TNF- $\alpha$ and IL-1 $\beta$ (Fig. $2 \mathrm{~A}$ and $2 \mathrm{~B}$ ). Knockdown of miR-874$3 p$ aggravated LPS-stimulated inflammation in HPAEpiC 
(Fig. 2A and 2B), suggesting that miR-874-3p could attenuate LPS-induced inflammation in HPAEpiC.

\section{MiR-874-3p attenuated LPS-induced apoptosis in HPAEpiC}

LPS decreased viability of HPAEpiC compared to the control (Fig. 3A). Transfection with miR-874-3p in LPS-treated HPAEpiC increased viability compared to NC mimic (Fig. 3A), while transfection with miR-874$3 \mathrm{p}$ inhibitor decreased viability compared to $\mathrm{NC}$ inhibitor (Fig. 3A). Similarly, LPS stimulated apoptosis of HPAEpiC (Fig. 3B and 3C) with reduction of $\mathrm{Bcl}-2$ and increase of Bax and cleaved caspase-3 (Fig. 3D) compared to the control. However, transfection with miR874-3p mimic in LPS-treated HPAEpiC suppressed apoptosis (Fig. 3B and 3C) with increase of Bcl-2 and reduction of Bax and cleaved caspase-3 (Fig. 3D). Knockdown of miR-874-3p aggravated LPS-stimulated apoptosis in HPAEpiC (Fig. 3B and 3C). Protein expression of $\mathrm{Bcl}-2$ was down-regulated, while Bax and cleaved caspase-3 were up-regulated in LPS-treated HPAEpiC transfected with miR-874-3p inhibitor (Fig. 3D), suggesting that miR-874-3p could attenuate LPS-induced apoptosis in HPAEpiC.

\section{Binding between miR-874-3p and EGR3}

TargetScan analysis (http://www.targetscan.org/ vert_71/) was used to identify the downstream target of miR-874-3p and EGR3 was predicted to bind with
miR-874-3p (Fig. 4A). Over-expression of miR-874$3 p$ decreased luciferase activity of EGR3-WT (Fig. 4B), while knockdown of miR-874-3p increased the activity (Fig. 4B). However, mutation at the binding site between miR-874-3p and EGR3 blocked the effect of miR-874-3p on luciferase activity of EGR3-MUT (Fig. $4 \mathrm{~B})$, suggesting that miR-874-3p could direct bind to EGR3. mRNA (Fig. 4C) and protein (Fig. 4D) expressions of EGR3 were reduced by miR-874-3p mimic, while knockdown of miR-874-3p increased EGR3 expression (Fig. 4C and 4D). EGR3 was dose-dependently increased in LPS-treated HPAEpiC (Fig. 4E), suggesting negative correlation with miR-874-3p and potential role of miR-874-3p/EGR3 in pediatric pneumonia.

EGR3 over-expression reversed the suppressive effects of miR-874-3p on inflammation and apoptosis in LPSinduced HPAEpiC

To investigate the role of miR-874-3p/EGR3 in pediatric pneumonia, HPAEpiC were co-transfected with pcDNA-EGR3 and miR-874-3p mimic, and then incubated with $10 \mathrm{mg} / \mathrm{mL}$ LPS. miR-874-3p over-expression reduced LPS-induced EGR3 expression (Fig. 5A), while co-transfection with miR-874-3p mimic and pcDNAEGR3 reversed the suppressive effect of miR-874-3p on EGR3 expression (Fig. 5A). EGR3 over-expression aggravated LPS-induced increase of TNF- $\alpha$ and IL-1 $\beta$ (Fig. 5B), decrease of cell viability (Fig. 5C) and increase of apoptosis (Fig. 5D). However, over-expression of miR-
A

$\begin{array}{ll}\text { EGR3-WT } & 5^{\prime} \text {...UCUUUCCACGACCCCCAGGGCAA...3' } \\ \text { hsa-miR-874-3p } & \text { 3' AGCCAGGGAGCCCGGUCCCGUC 5' }\end{array}$

EGR3-MUT $\quad 5$ '...UCUUUCCACGACCCCGGUCCCGUA...3'
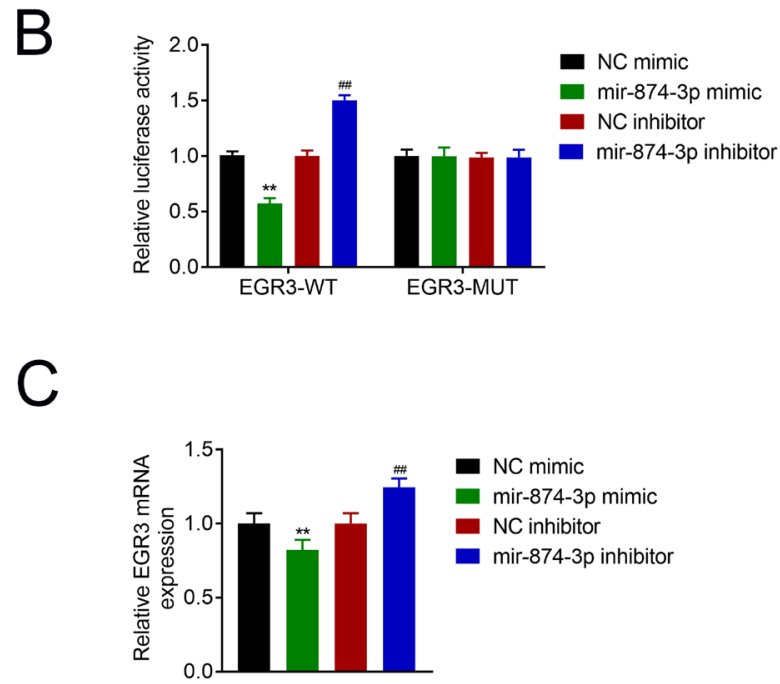
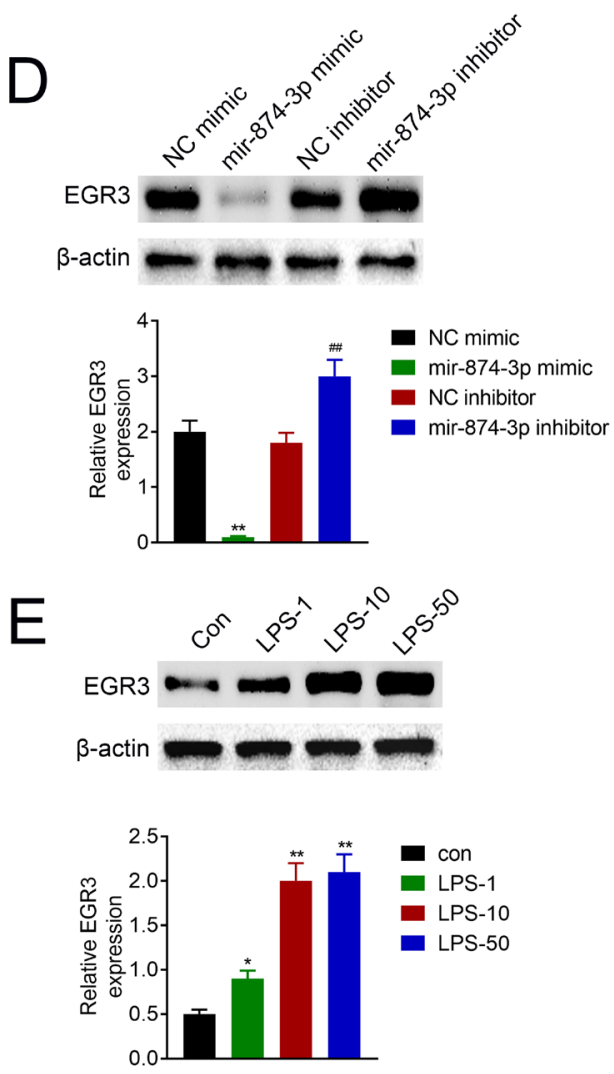

Figure 4. Binding between miR-874-3p and EGR3

(A) Potential binding sites between miR-874-3p and EGR3.

Over-expression of miR-874-3p decreased luciferase activity of EGR3-WT, while knockdown of miR-874-3p increased luciferase activity of EGR3-WT. However, mutation at the binding site between miR-874-3p and EGR3 blocked the effect of miR-874-3p on luciferase activity of EGR3-MUT. (B) mRNA expression of EGR3 was reduced by miR-874-3p mimic, while knockdown of miR-874-3p increased EGR3 expression. (C) Protein expression of EGR3 was reduced by miR-874-3p mimic, while knockdown of miR-874-3p increased EGR3 expression. (D) EGR3 was dose-dependently increased in LPS-induced HPAEpiC. ${ }^{*} p<0.05,{ }^{* * * * \# p} p<0.01$. 

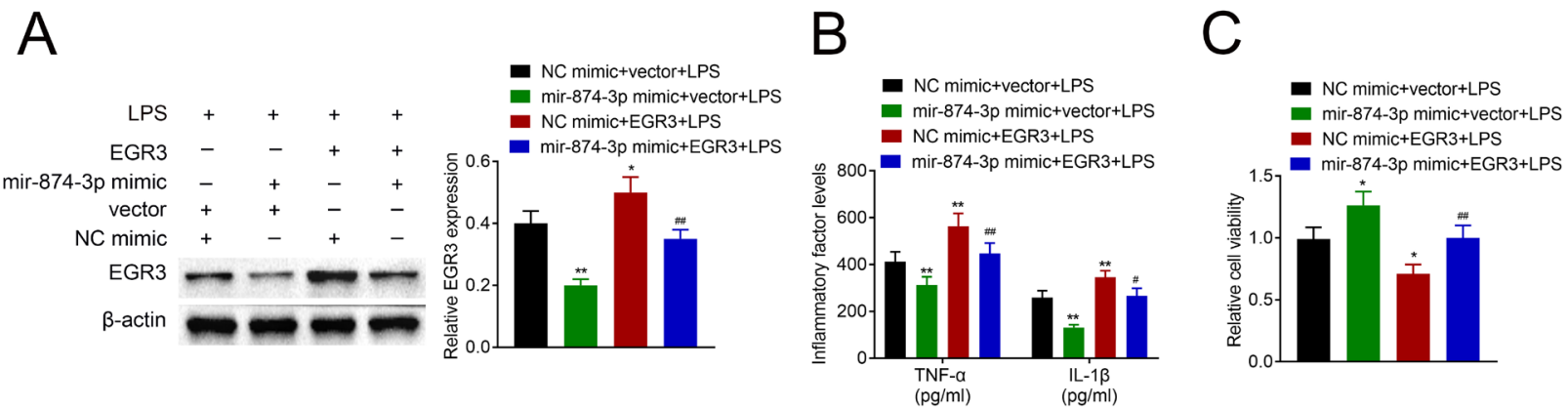

D
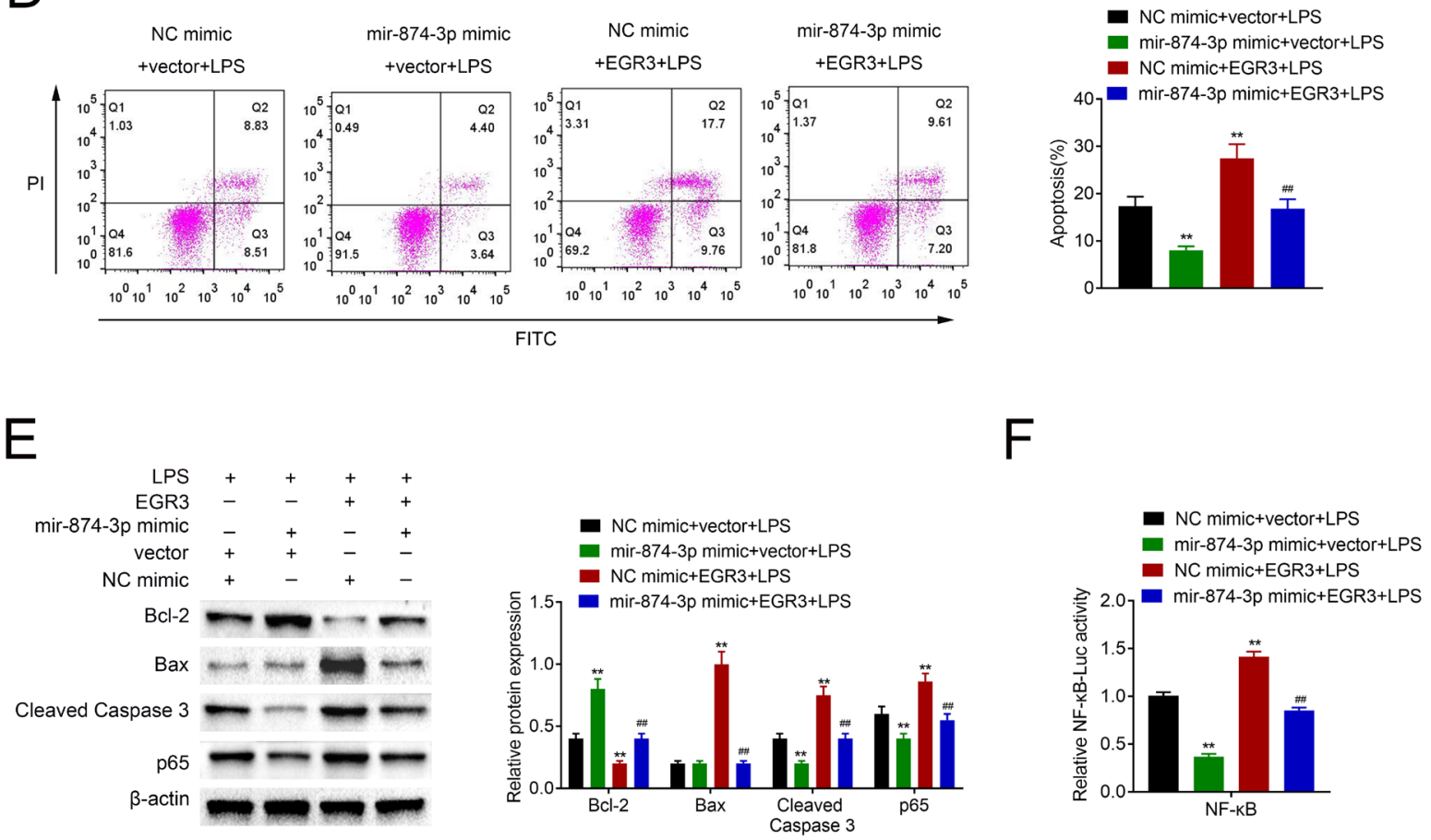

Figure 5. EGR3 over-expression counteracted the suppressive effects of miR-874-3p on inflammation and cell apoptosis in LPS-treated HPAEpiC

(A) Over-expression of miR-874-3p decreased LPS-induced EGR3 expression, while co-transfection with miR-874-3p mimic and pcDNAEGR3 reversed the suppressive effect of miR-874-3p on EGR3 expression. (B) EGR3 over-expression aggravated LPS-induced increase of TNF- $\alpha$ and IL-1 $\beta$, over-expression of miR-874-3p attenuated EGR3-induced increase of TNF- $\alpha$ and IL-1 $\beta$. (C) EGR3 over-expression aggravated LPS-induced decrease of cell viability, over-expression of miR-874-3p attenuated EGR3-induced decrease of cell viability. (D) EGR3 over-expression aggravated LPS-induced increase of cell apoptosis, over-expression of miR-874-3p attenuated EGR3-induced increase of cell apoptosis. (E) EGR3-induced decrease of $\mathrm{Bcl}-2$, increase of Bax, cleaved caspase-3 and p65 were reversed by miR-874-3p mimic.

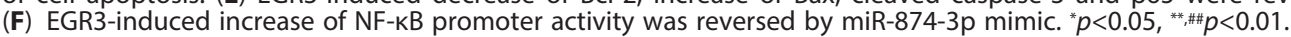

874-3p attenuated EGR3-induced increase of TNF- $\alpha$ and IL-1 $\beta$ (Fig. 5B), decrease of cell viability (Fig. 5C) and increase of apoptosis (Fig. 5D) in LPS-induced HPAEpiC. Similarly, EGR3-induced reduction of Bcl-2, increase of Bax and cleaved caspase- 3 were also reversed by miR874-3p mimic (Fig. 5E). Over-expression of miR-874-3p reduced p65 expression (Fig. 5E) and NF- $x \mathrm{~B}$ promoter activity (Fig. 5F) in LPS-induced HPAEpiC, while EGR3 over-expression enhanced p65 expression (Fig. 5E) and $\mathrm{NF}-x \mathrm{~B}$ promoter activity (Fig. $5 \mathrm{~F}$ ) and reversed the suppressive effects of miR-874-3p on NF- $x \mathrm{~B}$ pathway (Fig. 5E and 5F). These results suggested that miR-874$3 \mathrm{p}$ could repress LPS-induced inflammation and apoptosis in HPAEpiC via negative regulation of EGR3 and inhibition of $\mathrm{NF}-x \mathrm{~B}$ pathway.

\section{DISCUSSION}

Alveolar epithelial cells are the prime sites of bacterial or viral infection, and the infection could result in apoptosis and cytotoxicity of the epithelial cells (Herold et al., 2008). Prevention of apoptosis and cytotoxicity of alveolar epithelial cells could help ameliorate pneumonia (Kosmider et al., 2012). MiRNAs, with regulatory ability in cellular senescence, have been shown to be implicated in apoptosis of alveolar epithelial cells (Adameova et al., 2018). Since miR-874 has been reported to suppress cell proliferation and promote non-small cell lung cancer cell apoptosis (Wang et al., 2020), the potential regulatory roles of miR-874-3p in cytotoxicity of alveolar epithelial cells and development of pneumonia were evaluated in this study. 
MiR-874-3p was down-regulated in serum of patients with pneumonia and LPS-treated HPAEpiC. LPS, as an endotoxin, could stimulate apoptosis and cytotoxicity. Therefore, LPS was widely used as a stimulatory model of acute pneumonia (Zhang et al., 2020). Our results indicated that LPS treatment reduced viability of HPAEpiC and enhanced apoptosis. Moreover, LPS could stimulate inflammatory response and promote pathogenesis of pneumonia (Meng et al., 2012). Proinflammatory factors TNF- $\alpha$ and IL-1 $\beta$ were found to be up-regulated in HPAEpiC post LPS treatment during this study. Alleviation of LPS-induced cytotoxicity and inflammation could help improve acute pneumonia (Zhang et al., 2019). We therefore validated the role of miR-874-3p in LPS-induced inflammation and apoptosis of HPAEpiC.

In line with previous study that miR-874 suppressed cell proliferation and promoted non-small cell lung cancer cell apoptosis (Wang et al., 2020), knockdown of miR-874-3p aggravated LPS-induced decrease of cell viability and increase of apoptosis in HPAEpiC. Moreover, LPS-induced secretion of TNF- $\alpha$ and IL-1 $\beta$ was also repressed by miR-874-3p over-expression, consistent with previous study that miR-874 could attenuate inflammatory response with decreased IL- $1 \beta$ and $\mathrm{TNF}-\alpha$ in diabetic nephropathy (Yao et al., 2019). These results suggested that miR-874-3p could alleviate LPS-induced inflammation and apoptosis in alveolar epithelial cells, thus ameliorating development of pediatric pneumonia. Animal model of pneumonia should be established to further investigate the role of miR-874-3p in pediatric pneumonia.

MiR-874 could regulate necrosis through caspase-8 (Wang et al., 2013), and attenuate inflammatory response by toll-like receptor-4 (Yao et al., 2019). Our data demonstrated that miR-874-3p bind to EGR3. EGR3 was reported to promote inflammatory response in prostate cancer by increasing IL-6 and IL-8 (Baron et al., 2015). Our results showed that EGR3, increased in HPAEpiC after LPS treatment, counteracted the suppressive effects of miR-874-3p on inflammation and apoptosis in LPS-treated HPAEpiC. Therefore, miR-874-3p repressed LPS-induced apoptosis and inflammation in HPAEpiC via negative regulation of EGR3. EGR1 was found to be a neuronal target of $\mathrm{NF}-\varkappa \mathrm{B}$ during Alzheimer's disease (Snow \& Albensi 2016). EGR3 could interact with $\mathrm{NF}-x \mathrm{~B}, \mathrm{p} 50$, and p65 (Wieland et al., 2005), and promote activation of NF-xB pathway (Kurosaka et al., 2017). Our data revealed that over-expression of miR-874-3p reduced $\mathrm{p} 65$ expression and $\mathrm{NF}-x \mathrm{~B}$ promoter activity in LPS-treated HPAEpiC, while EGR3 over-expression enhanced p65 expression and NF- $x \mathrm{~B}$ promoter activity and reversed the suppressive effects of miR-874-3p on $\mathrm{NF}-x \mathrm{~B}$ pathway. Activation of NF- $x \mathrm{~B}$ could contribute to development of pneumonia (Jones et al., 2006), inhibition of NF- $x \mathrm{~B}$ pathway could protect against LPSinduced apoptosis and inflammation of alveolar epithelial cells (Shao et al., 2017; Cheng et al., 2020), and attenuate pediatric pneumonia (Liu et al., 2017; Li et al., 2019). Therefore, the suppressive effect of miR-874-3p in LPSinduced inflammation and apoptosis of HPAEpiC might be dependent on inactivation of EGR3/NF- $x \mathrm{~B}$ pathway.

\section{CONCLUSION}

MiR-874-3p was reduced in serum of pediatric patients with pneumonia. We found that miR-874-3p could promote viability of LPS-treated alveolar epithelial cells, while suppressing apoptosis and inflammation through targeting the EGR3/NF- $x \mathrm{~B}$ pathway. This study provided a potential therapeutic target for pediatric pneumonia.

\section{Acknowledgements}

Not applicable.

\section{Funding}

Not applicable.

\section{Competing interests}

The authors state that there are no conflicts of interest to disclose.

\section{Ethics approval}

This study was approved by the Ethics Committee of Zhejiang Zhoushan Putuo District Hospital of Traditional Chinese Medicine.

\section{Statement of Informed Consent}

Written informed consent was obtained from a legally authorized representative(s) for anonymized patient information to be published in this article.

\section{Availability of data and materials}

All data generated or analyzed during this study are included in this published article.

\section{Authors' contributions}

Huirun Yang and Yang Dong designed the study, supervised data collection, Yan Zhou analyzed the data, interpreted the data, Huajun Li prepare the manuscript for publication and reviewed the draft of the manuscript. All authors have read and approved the manuscript.

\section{REFERENCE}

Adameova AD, Bhullar SK, Elimban V, Dhalla NS (2018) Activation of $\beta 1$-adrenoceptors may not be involved in arrhythmogenesis in ischemic heart disease. Revi Cardiovasc Med 19: 97-101. https://doi. org/10.31083/j.rcm.2018.03.3181

Alcoba G, Keitel K, Maspoli V, Lacroix L, Manzano S, Gehri M, Tabin R, Gervaix A, Galetto-Lacour A (2017) A three-step diagnosis of pediatric pneumonia at the emergency department using clinical predictors, C-reactive protein, and pneumococcal PCR. Eur J Pediatric 176: 815-824. https://doi.org/10.1007/s00431-017-2913-0

Baron VT, Pio R, Jia Z, Mercola D (2015) Early Growth Response 3 regulates genes of inflammation and directly activates IL6 and IL8 expression in prostate cancer. Brit J Cancer 112: 755-764. https:// doi.org/10.1038/bjc.2014.622

Cheng D, Zhu C, Liang Y, Xing Y, Shi C (2020) MiR-424 overexpression protects alveolar epithelial cells from LPS-induced apoptosis and inflammation by targeting FGF2 via the NF- $x \mathrm{~B}$ pathway. Life Sci 242: 117213. https://doi.org/https://doi.org/10.1016/j. lfs.2019.117213

Cho SJ, Kang MJ, Homer RJ, Kang HR, Zhang X, Lee PJ, Elias JA, Lee CG (2006) Role of early growth response-1 (Egr-1) in interleukin-13-induced inflammation and remodeling. I Biol Chem 281: 8161-8168. https://doi.org/10.1074/jbc.M506770200

Choi HJ, Kim J, Park SH, Do KH, Yang H, Moon Y (2012) Proinflammatory $\mathrm{NF}-x \mathrm{~B}$ and early growth response gene 1 regulate epithelial barrier disruption by food additive carrageenan in human intestinal epithelial cells. Toxicol Lett 211: 289-295. https://doi. org/10.1016/j.toxlet.2012.04.012

Guillot L, Nathan N, Tabary O, Thouvenin G, Le Rouzic P, Corvol H, Amselem S, Clement A (2013) Alveolar epithelial cells: Master regulators of lung homeostasis. Int J Biochem Cell Biol 45: 2568-2573. https://doi.org/https://doi.org/10.1016/j.biocel.2013.08.009

Herold S, Steinmueller M, von Wulffen W, Cakarova L, Pinto R, Pleschka S, Mack M, Kuziel WA, Corazza N, Brunner T, Seeger W, Lohmeyer J (2008) Lung epithelial apoptosis in influenza virus pneumonia: the role of macrophage-expressed TNF-related 
apoptosis-inducing ligand. J Exp Med 205: 3065-3077. https://doi. org/10.1084/jem.20080201

Huang F, Zhang J, Yang D, Zhang Y, Huang J, Yuan Y, Li X, Lu G (2018) MicroRNA expression profile of whole blood is altered in adenovirus-infected pneumonia children. Med Inflamm 2018: 2320640-2320640. https://doi.org/10.1155/2018/2320640

Huang S, Feng C, Zhai YZ, Zhou X, Li B, Wang LL, Chen W, Lv FQ, Li TS (2017) Identification of miRNA biomarkers of pneumonia using RNA-sequencing and bioinformatics analysis. Exp Ther Med 13: 1235-1244. https://doi.org/10.3892/etm.2017.4151

Ito Y, Correll K, Zemans RL, Leslie CC, Murphy RC, Mason RJ (2015) Influenza induces IL-8 and GM-CSF secretion by human alveolar epithelial cells through HGF/c-Met and TGF- $\alpha /$ EGFR signaling. Am J Physiol Lung Cell Mol Physiol 308: L1178-L1188. https:// doi.org/10.1152/ajplung.00290.2014

Jiang D, Sun X, Wang S, Man H (2019) Upregulation of miR-874-3p decreases cerebral ischemia/reperfusion injury by directly targeting BMF and BCL2L13. Biomed Pharmacother 117: 108941. https://doi. org/https://doi.org/10.1016/j.biopha.2019.108941

Jones MR, Simms BT, Lupa MM, Kogan MS, Mizgerd JP (2006) Lung NF-kappaB activation and neutrophil recruitment require IL-1 and TNF receptor signaling during pneumococcal pneumonia. I Immunol 175: 7530-7535. https://doi.org/10.4049/jimmunol.175.11.7530

Kosmider B, Messier EM, Janssen WJ, Nahreini P, Wang J, Hartshorn KL, Mason RJ (2012) Nrf2 protects human alveolar epithelial cells against injury induced by influenza A virus. Respir Res 13: 43-43. https://doi.org/10.1186/1465-9921-13-43

Kurosaka M, Ogura Y, Funabashi T, Akema T (2017) Early growth response 3 (Egr3) contributes a maintenance of C2C12 myoblast proliferation. J Cell Physiol 232: 1114-1122. https://doi.org/10.1002/ jcp. 25574

Li J, Liu S (2020) LncRNA GAS5 suppresses inflammatory responses and apoptosis of alveolar epithelial cells by targeting miR-429/ DUSP1. Exp Mol Pathol 113: 104357. https://doi.org/https://doi. org/10.1016/j.yexmp.2019.104357

Li S, Cui W, Song Q, Zhou Y, Li J (2019) miRNA-302e attenuates inflammation in infantile pneumonia though the RelA/BRD4/ NF- $x \mathrm{~B}$ signaling pathway. Int J Mol Med 44: 47-56. https://doi. org/10.3892/ijmm.2019.4194

Li S, Miao T, Sebastian M, Bhullar P, Ghaffari E, Liu M, Symonds AL, Wang P (2012) The transcription factors Egr2 and Egr3 are essential for the control of inflammation and antigen-induced proliferation of B and T cells. Immunity 37: 685-696. https://doi. org/10.1016/j.immuni.2012.08.001

Liu Z, Yu H, Guo Q (2017) MicroRNA-20a promotes inflammation via the nuclear factor- $x \mathrm{~B}$ signaling pathway in pediatric pneumonia. Mol Med Rep 17: 612-617. https://doi.org/10.3892/mmr.2017.7899

Meng N, Zhao J, Su L, Zhao B, Zhang Y, Zhang S, Miao J (2012) A butyrolactone derivative suppressed lipopolysaccharide-induced autophagic injury through inhibiting the autoregulatory loop of $\mathrm{p} 8$ and p53 in vascular endothelial cells. Int I Biochem Cell Biol 44: 311-319. https://doi.org/https://doi.org/10.1016/j.biocel.2011.11.001

Miao T, Symonds ALJ, Singh R, Symonds JD, Ogbe A, Omodho B, Zhu B, Li S, Wang P (2017) Egr2 and 3 control adaptive immune responses by temporally uncoupling expansion from $\mathrm{T}$ cell differentiation. J Exp Med 214: 1787-1808. https://doi.org/10.1084/ jem.20160553

Qinghe G, Guangyu L, Xiaobin L, Qingsong H (2019) Baicalein exerts a protective role in pneumonia caused by Streptococcus pneumoniae. Frontiers Biosci (Landmark Edn) 24: 849-858. PMID: 30844717

Robb CT, Regan KH, Dorward DA, Rossi AG (2016) Key mechanisms governing resolution of lung inflammation. Semin Immunopathol 38: 425-448. https://doi.org/10.1007/s00281-016-0560-6

Shao L, Meng D, Yang F, Song H, Tang D (2017) Irisin-mediated protective effect on LPS-induced acute lung injury via suppressing inflammation and apoptosis of alveolar epithelial cells. Biochemi Biophys Res Commun 487: 194-200. https://doi.org/https://doi. org/10.1016/j.bbrc.2017.04.020

Snow WM, Albensi BC (2016) Neuronal gene targets of NF- $x \mathrm{~B}$ and their dysregulation in Alzheimer's disease. Frontiers Mol Neurosci 9: 118-118. https://doi.org/10.3389/fnmol.2016.00118

Wang K, Liu F, Zhou LY, Ding SL, Long B, Liu CY, Sun T, Fan YY, Sun L, Li PF (2013) miR-874 regulates myocardial necrosis by targeting caspase-8. Cell Death Dis 4: e709-e709. https://doi. org $/ 10.1038 /$ cddis.2013.233

Wang S, Wu Y, Yang S, Liu X, Lu Y, Liu F, Li G, Tian G (2020) miR-874 directly targets AQP3 to inhibit cell proliferation, mobility and EMT in non-small cell lung cancer. Thoracic Cancer 11: 15501558. https://doi.org/10.1111/1759-7714.13428

Wieland GD, Nehmann N, Müller D, Eibel H, Siebenlist U, Sühnel J, Zipfel PF, Skerka C (2005) Early growth response proteins EGR-4 and EGR-3 interact with immune inflammatory mediators NF- $x$ B p50 and p65. J Cell Sci 118: 3203. https://doi.org/10.1242/jcs.02445

Xie K, Cai Y, Yang P, Du F, Wu K (2020) Upregulating microRNA874-3p inhibits CXCL12 expression to promote angiogenesis and suppress inflammatory response in ischemic stroke. Am J Physiol Cell Physiol 319: C579-C588. https://doi.org/10.1152/ajpcell.00001.2020

Yao T, Zha D, Gao P, Shui H, Wu X (2019) MiR-874 alleviates renal injury and inflammatory response in diabetic nephropathy through targeting toll-like receptor-4. J Cell Physiol 234: 871-879. https://doi. org/10.1002/jcp.26908

Zhang J, Mao F, Zhao G, Wang H, Yan X, Zhang Q (2020) Long non-coding RNA SNHG16 promotes lipopolysaccharides-induced acute pneumonia in A549 cells via targeting miR-370-3p/IGF2 axis. Int Immunopharmacol 78: 106065. https://doi.org/https://doi. org/10.1016/j.intimp.2019.106065

Zhang Y, Zhu Y, Gao G, Zhou Z (2019) Knockdown XIST alleviates LPS-induced WI-38 cell apoptosis and inflammation injury via targeting miR-370-3p/TLR4 in acute pneumonia. Cell Biochem Funct 37: 348-358. https://doi.org/10.1002/cbf.3392 